\title{
CÉLULAS-TRONCO E NEUROGÊNESE
}

\author{
Stem cells and neurogenesis
}

\author{
Luiz Roberto Tavares Filho ${ }^{1}$ \\ Fábio Rueda Faucz ${ }^{2}$
}

\section{Resumo}

O estudo de células-tronco se tornou um campo fascinante na área científica que cada vez mais vem atraindo e causando motivações em pesquisadores de todo o mundo, e suas aplicações tornaram as células tronco um dos campos mais pesquisados nos últimos anos. As células-tronco podem ser retiradas principalmente da medula óssea de indivíduos adultos ou do cordão umbilical, mas é no embrião ou no zigoto em desenvolvimento que elas possuem capacidade única de diferenciação em todas as células do corpo, e são chamadas "células-tronco embrionárias". O estudo da neurogênese no indivíduo adulto é outra área em que muitos estudos estão sendo realizados. Até algum tempo atrás, a neurogênese era somente aceita na fase de desenvolvimento embrionário, ou seja, durante a geração do feto e pouco depois no período pós-natal, onde todos os neurônios do indivíduo seriam formados, por esse motivo, falar em neurogênese no adulto era para muitos cientistas um absurdo. O primeiro relato de neurogênese no adulto data de 40 anos atrás, porém somente agora esta sendo aceita. Existem hoje várias opiniões sobre o assunto, algumas delas expressas neste trabalho. Palavras-chave: Células-tronco; Neurogênese; Sistema Nervoso Central.

\section{Abstract}

The study of stem cells became a fascinating field in the scientific area that more and more is attracting and causing motivations in researchers all over the world, in the last years its applications turned the stem cells one of the most researched fields. The stem cells can be removed mainly from the adult bone marrow or from the umbilical cord, but it is in the embryo or in the developing zygote that they possess unique capacity of differentiation in all body cells, and they are called "embryonic stem cells". The study of the neurogenesis in the adult individual is another area in which many studies are being accomplished. Some time ago the neurogenesis was only accepted in the embryonic developing phase, in other words, during the fetus generation and a little later, in the post-native period when all of the individual's neurons would be formed, for that reason, speaking in adult neurogenesis was, for many scientists, an absurd. The first adult neurogenesis report dates from 40 years ago, but just now this is being accepted. Today exist a lot of opinions on this subject, some of them are expressed in this paper.

Keywords: Stem cells; Neurogenesis; Central nervous system.

1 Curso de Especialização em Genética Humana. Pontifícia Universidade Católica do Paraná, 2004.

E-mail: luiz_filho@click21.com.br

2 Professor da Pontifícia Universidade Católica do Paraná - CCBS. Endereço para correspondência: Pontifícia Universidade Católica do Paraná - Centro de Ciências Biológicas e da Saúde, Rua Imaculada Conceição, 1155. Prado Velho, CEP 80215-901. Curitiba - PR.

E-mail: fruedas@onda.com.br 


\section{Introdução}

Poucos assuntos na ciência biomédica capturaram a imaginação da comunidade científica e do público como tem sido o uso de célulastronco (CT) para o reparo de tecidos danificados, pois trazem a chance de poderem substituir as células com atrofia ou até as que foram perdidas inteiramente durante uma lesão. O aparecimento das células-tronco é a esperança da restauração da função celular e do tecido em questão. Além do reparo do tecido, as células-tronco cultivadas puderam também encontrar aplicação nas análises de mecanismos das doenças e do desenvolvimento normal, e como veículos para a terapia do gene (EVERS et al., 2003).

As pesquisas sobre CT estão avançando no conhecimento sobre como uma célula pode substituir células danificadas por muitas das doenças que nos afetam ou por lesões causadas em acidentes. Esta área promissora da ciência está conduzindo também os cientistas a investigar a possibilidade de terapias celulares para regeneração ou reparação de uma doença. Mas como muitos campos em expansão do inquérito científico, a pesquisa sobre CT levanta perguntas tão rapidamente como geram novas descobertas (COOPER, 2004). Como este tipo de célula parece apresentar baixos problemas de rejeição, elas hoje são alvo de estudos para a tentativa de cura de diversas doenças. Sua função é ajudar no reparo de lesões, e é a esperança para a cura de doenças degenerativas, como Alzheimer e Parkinson (ZATZ, 2004).

Um outro campo fascinante é o estudo da neurogênese no indivíduo adulto, onde as CT são o foco central. Até pouco tempo atrás se acreditava que os neurônios não podiam ser gerados no indivíduo adulto; hoje as pesquisas indicam que existe a formação de novos neurônios e que esses podem estar envolvidos na aprendizagem e na formação de traços de memória (ALBERG et al., 2000).

Neurogênese é um processo que envolve proliferação, migração e diferenciação celular. Durante a proliferação, as CT neurais se dividem assimetricamente, sendo que uma permanece indiferenciada e a outra célula progenitora neuronal pode se multiplicar e se diferenciar. De maneira geral, o processo de diferenciação transforma uma célula imatura ainda sem comprometimento em células neuronais ou células da glia maduras (SHI et al., 2004, MARKAKIS et al., 2004).

A primeira evidência de que o cérebro do adulto continha algumas células que se dividiam, proliferavam e que possuíam fenótipo de neurônios foi relatada por Altman na década de 60 (ALTMAN; DAS, 1965;1966). As investigações subseqüentes forneceram mais evidências para sustentação da neurogênese no adulto (GOLDMAN; NOTTEBOHM, 1983; KAPLAN; HINDS, 1977; NOTTEBOHM, 1981). A neurogênese no adulto foi observada em diversas espécies, tais como o rato (ALTMAN; DAS, 1965, 1966; KAPLAN; HINDS, 1977; GOULD et al., 1992), coelho (GUENEAU et al., 1982), gato (WYSS; SRIPANIDKULCHAI, 1985), rato (KEMPERMANN et al., 1997), nos primatas (GOULD et al., 1999a; KORNACK; RAKIC, 1999) e também no cérebro humano (ERIKSSON et al., 1998).

\section{Revisão Bibliográfica}

Uma CT tem habilidade de se dividir durante toda vida do organismo e sob circunstâncias adequadas ou estímulos específicos podem se diferenciar em diferentes tipos de células. Isto é, as CT têm o potencial de tornarem-se células maduras com formas características e funções especializadas, tais como células coração, neurônios, entre outras (SLACK, 2002).

Existem algumas teorias sobre a divisão das CT (FURUSAWA; KANEKO, 2001; DEENICK et al., 2003). Uma dessas postula que a CT é definida por duas propriedades: primeiro, é uma célula capaz de se dividir indefinidamente, produzindo uma população de prole idêntica; e em segundo, pode submeter-se a uma divisão assimétrica para produzir duas células filhas diferentes. Uma é idêntica à mãe e continua a contribuir com a linha original indiferenciada. A outra possui instruções genéticas diferentes e é caracterizada por uma capacidade proliferativa reduzida e por um potencial de diferenciação mais restringido do que sua mãe. Eventualmente, uma célula-tronco torna-se uma célula "progenitora" ou "precursora", cometida a produzir um ou mais tipos de células totalmente diferenciadas tais como os neurônios ou as células musculares (RAFF, 2003; WAGERS; WEISSMAN, 2004).

Durante a fertilização, quando o esperma e o óvulo juntam-se, é produzida uma célula 
que se multiplica e é capaz de dar origem a um organismo inteiro. As células embrionárias são classificadas como totipotentes, que significa que esta célula tem potencial ilimitado de diferenciação. Por um tempo curto, cada divisão do zigo to cria células totipotentes idênticas chamadas blastômeros. Chegando ao quarto dia de desenvolvimento embrionário, as células totipotentes começam a especializar-se, dando forma ao blastocisto. A camada exterior de células, sinciciotrofoblasto, dá origem a maior parte da placenta e aos anexos extra-embrionários necessários à formação do feto. $\mathrm{O}$ conjunto interno de células, citotrofoblasto, origina 0 disco embrionário contendo todos os tecidos do corpo humano. Embora essas células da massa celular interna se diferenciem em cada tipo de tecido no corpo, não podem formar a placenta ou outros anexos extra-embrionários, devido a isso são classificadas como pluripotentes. Essas células continuam a se especializar, transformam-se em células que conduzem somente ao desenvolvimento de tecidos específicos. Algumas células indiferenciadas ficarão confinadas a medula óssea, enquanto outras estarão presentes em outros tecidos. Estas últimas carregam esta especialização extra, são consideradas multipotentes (ELUS et al., 2004). As células multipotentes podem ainda ser encontradas durante a vida adulta de uma pessoa. Embora encontradas geralmente em quantidades pequenas, as CT do adulto são de muita importância na sustentação da vida. Por exemplo, as células vermelhas do sangue são substituídas continuamente, e a produção das novas células vermelhas é iniciada por uma CT hematopoiética, que também gera as de origem linfóide.

As CT embrionárias são células pluripotentes derivadas da massa celular interna do blastocisto, que podem ser propagadas indefinidamente em condições controladas de laboratório. As CT embrionárias diferenciam-se em todas as linhagens de células provenientes dos três folhetos embrionários. Embora as CT embrionárias sejam isoladas de óvulos humanos fecundados ou via transferência de núcleo somático (THOMSON et al., 1998), seu uso na pesquisa assim como na terapêutica era impedido por considerações éticas (FRANKEL, 2000).

A presença das CT já está confirmada em tecidos como medula óssea (WEISSMAN, 2000), sistema nervoso (GAGE, 2000), digestório (POTTEN, 1998), epitelial (WATT, 1997), hepá- tico (ALISON; SARRAF, 1998), e a maioria possui caracterização por marcadores específicos de superfície.

\section{Neurogênese}

Durante a última década imaginou-se que o sistema nervoso do adulto teria o potencial de se regenerar após ferimento ou doença.

Durante todo o século 20, os protocolos de terapias visando à regeneração neural focalizou-se em manter os neurônios vivos após o trauma. À exceção de alguns estudos executados na década de 60, que sugeriram que a proliferação de células no cérebro do adulto daria origem aos novos neurônios e a glia (ALTMAN; DAS, 1965; ALTMAN, 1969), a literatura dizia que os neurônios eram produzidos somente durante a embriogênese no sistema nervoso central (SNC). Este dogma provou estar incorreto e é agora evidente que os novos neurônios são gerados em cérebros maduros de ratos (LUSKIN, 1993; LOIS; ALVAREZ, 1994) e primatas (GOULD et al., 1999b).

Aproximadamente 40 anos após seu primeiro relato, a adição de novos neurônios ao cérebro de mamíferos adultos foi aceita apenas recentemente (LENNINGTON et al., 2003).

Uma das dificuldades em estudos da neurogênese em adultos era a incerteza se as células adultas geradas eram glia ou neurônios. No cérebro do adulto alguns progenitores formam os neurônios e a glia (ALVAREZ-BUYLA et al., 2001). De fato, evidências recentes em ratos adultos indicam que os neurônios novos do granule se formam de células com características gliais (SERI et al., 2001). Na década de 90 foram desenvolvidos alguns trabalhos que finalmente deram início à caracterização da neurogênese no adulto. $\mathrm{O}$ primeiro foi por meio da introdução de marcadores específicos da célula para a identificação imunohistoquímica do fenótipo de células recentemente geradas (CAMERON; GOULD, 1994; OKANO et al., 1993).

O cérebro continua a produzir os novos neurônios durante toda a vida do indivíduo (NOTTEBOHM, 1989). No hipocampo de rato, milhares são produzidos diariamente. A aprendizagem associativa pode realçar sua sobrevivência (GOULD et al., 1999c). Entretanto, até o momento, nenhum trabalho conseguiu confirmar a participação dos novos neurônios na formação da memória. A redução na neurogênese não induziu a 
morte dos neurônios hipocampais maduros nem alterou permanentemente propriedades neuropsicológicas da região. Além disso, a recuperação da produção da célula foi associada com a habilidade de adquirir traços de memória (MINGER; DAVIES, 1999). Estes resultados indicam que os neurônios recentemente gerados no adulto não apenas são afetados pela formação de uma memória hipocampal-dependente, mas participam também dela (GOULD et al., 1999d).

A participação da formação hipocampal na aprendizagem e na memória foi reconhecida por décadas (MILNER, 1972; SQUIRE, 1982), mas apesar dos anos de pesquisa intensiva, virtualmente nada é sabido sobre a base biológica desta função. Uma série recente de descobertas sobre a formação hipocampal sugere uma nova aproximação à base celular da aprendizagem e da memória. Usando marcadores histológicos de proliferação de células, estes estudos demonstraram que a produção dos novos neurônios ocorre em uma variedade de mamíferos adultos, incluindo seres humanos.

Existem fatores que regulam a neurogênese hipocampal no adulto, estudos recentes identificaram um número de fatores que modulam a neurogênese hipocampal do adulto. Ao considerar estes fatores que regulam a produção do neurônio no adulto, é importante notar que a mudança no número de células novas provavelmente não apresenta conseqüências funcionais imediatas. Isto é particularmente verdadeiro quando somente a taxa de proliferação da célula é alterada, pois as células recém-geradas requerem 0 momento de se tornarem integradas funcionalmente aos circuitos existentes. Embora as mudanças funcionais não pudessem ser imediatamente evidentes, os fatores poderiam exercer efeitos significativos durante todo o curso de diferenciação para influenciar a função no prazo correto. Entretanto, estes fatores devem agir de forma relativamente rápida, a fim de se obter os efeitos funcionais significativos. As mudanças crônicas na produção e na sobrevivência da célula são capazes de exercer efeitos aditivos durante toda a produção (GOULD et al., 1999d).

As elevações nas concentrações de corticosterona in vitro diminuem o número de células proliferativas visto que a remoção de esteróides adrenais estimula a proliferação e produção de células (GOULD et al., 1992;
MOGHADDAM et al., 1994). A ação supressiva dos glicocorticóides na neurogênese é biologicamente relevante porque as circunstâncias que são associadas aos hormônios adrenais elevados, tais como o estresse (GOULD et al., 1997), são acompanhadas também pela produção diminuída da célula do granule em roedores e em primatas (GOULD et al., 1989; KUHN et al., 1996; KRUGERS et al., 1997). Além disso, as circunstâncias associadas à inibição prolongada da neurogênese, tais como o tratamento crônico com corticosterona, estresse crônico, entre outros, foram associadas com o desempenho diminuído no hipocampo dependente (GALAGHER; PELLEYMOUNTER, 1998). Em contraste aos efeitos supressivos do estresse e da corticosterona na proliferação de célula ao tratamento do estrógeno, foi mostrado como importante para realçar a proliferação da célula precursora. Inversamente, níveis baixos de estrógeno (uma circunstância sabidamente diminuidora da proliferação da célula precursora) foram ligados ao decline cognitivo associado à idade (GOULD et al., 1998).

As CT neurais são células multipotentes capazes se replicarem e se diferenciarem em neurônios, astrócitos ou em oligodendrocitos no sistema nervoso central (SNC) (TAUPIN; GAGE, 2002). Por causa de sua plasticidade e multipotencialidade intrínsecas, há grandes expectativas de que o transplante de células no SNC forneça finalmente benefícios imensos no tratamento das doenças neurodegenerativas (WEBBER; MINGER, 2004).

As CT são também responsáveis pela geração de outros sistemas somáticos durante o desenvolvimento, tal como o SNC. As CT neurais no SNC têm a habilidade de gerar neurônios, oligodendrocitos e astrócitos (LENNINGTON et al., 2004).

Pensava-se que o sistema nervoso adulto tinha habilidade muito limitada de se regenerar. A descoberta das CT neurais no adulto foi uma surpresa (ALVAREZ-BUYLA; TEMPLE, 1998; TEMPLE, 2001). Desde a observação inicial feita há 40 anos (ALTMAN, 1962), a neurogênese foi encontrada em regiões específicas do SNC de todos os mamíferos adultos examinados (MACKOWIAK et al., 2004), incluindo os seres humanos (LENNINGTON et al., 2003). A existência das CT neurais no SNC de adultos foi sugerida mais tarde pelo isolamento de CT neurais multipotentes do cérebro de ratos adul- 
tos (REYMONDS; WEISS, 1992). Na última década, CT neurais multipotentes foram isoladas de diversas regiões do SNC adulto dos roedores e seres humanos (REYMONDS; WEISS, 1992; SVENDSEN et al., 1999; NUNES et al., 2003). Estas CT neurais do adulto podem ser amplificadas in vitro sem perder sua multipotencialidade, sendo capazes de formar neurônios, astrócitos, e oligodendrocitos em cultura, e em seguida serem transplantadas para regiões específicas in vivo (PETERSON, 2004). Os rápidos avanços na biologia do SNC adulto levantaram grandes expectativas de que estas células podem ser usadas como potenciais recursos para a terapia neural de substituição após trauma ou relacionadas a doenças neurológicas degenerativas (BJORKLUND; LINDVALL, 2002; OSTENFELD; SVENDSEN, 2003; PETERSON, 2004; BLESCH; TUSZYNSKI, 2004; KIM et al., 2004; TOHIL; TERENG HI, 2004; WEBBER; MINGER, 2004; WESTPHAL; BLACK, 2004).

\section{Referências}

ABERG, M.A.; ABERG, N. D.; HEDBACKER, H.; O SCARSSO N, J.; ERIKSSON, P. S. Peripheral infusion of IGF-I selectively induces neurogenesis in the adult rat hippocampus. J. Neurosci., v. 20, p. 2896-2903, 2000.

ALISON, M.; SARRAF, C. Hepatic stem cells. J. Hepatol, v. 29, p. 678-683, 1998.

ALTMAN, J. Are neurons formed in the brains of adult mammals? Science, v. 135, p.1127-8, 1962.

ALTMAN, J.; DAS, G. D. Autoradiographic and histological evidence of postnatal hippocampal neurogenesis in rats. J. Comp Neurol, v. 124, p. 319-335, 1965.

ALTMAN, J.; DAS, G. D. Autoradiographic and histological studies of postnatal neurogenesis. I. A longitudinal investigation of the kinetics, migration and transformation of cells incorporating tritiated thymidine in neonate rats, with special reference to postnatal neurogenesis in some brain regions. J. Comp Neurol, v.126, p. 337-389, 1966.

ALTMAN, J. Autoradiographic and histological studies of post-natal neurogenesis IV. Cell proliferation and migration in anterior forebrain with special reference to persisting neurogenesis in olfactory bulb. J. Comp Neurol, v. 137, p. 433-457, 1969.

ALVAREZ-BUYLLA, A.; TEMPLE, S. Stem cells in the developing and adult nervous system. Proc. Natl. Acad. Sci., v. 96, p. 5263-5267, 1998.

ALVAREZ-BUYLA. A; GARCÍA-VERDUGO, J. M.; TRAMONTIN, A. D. A unified hypothesis on the lineage of neural stem cells. Nat. Rev. Neurosci., v. 2, p. 287-293, 2001.

BJO RKLUND, A.; LINDVALL, O. Cell replacement therapies for central nervous system disorders. Nat. Neurosci., v. 3, n. 6, p. 537-44, 2000.

BLESCH, A.; TUSZYNSKI, M. H. Gene therapy and cell transplantation for Alzheimer's disease and spinal cord injury. Yonsei Med. J., v. 30, n. 45 Suppl, p. 28-31, 2004.

CAMERON, H. A.; GOULD, E. Adult neurogenesis is regulated by adrenal steroids in the dentate gyrus. Neuroscience, v. 61, p. 203-209, 1994.

COOPER, M. Regenerative medicine: stem cells and the science of monstrosity. Med. Humanit., v.30, n. 1, p. 12-22, 2004.

DEENICK, E. K.; GETT, A. V.; HODGKIN, P. D. Stochastic model of $\mathrm{T}$ cell proliferation: a calculus revealing IL-2 regulation of precursor frequencies, cell cycle time, and survival. J. Immunol., v.170, n. 10, p. 4963-72, 2003.

ELLIS, P.; FAGAN, B. M.; MAGNESS, S. T.; HUTTON, S.; TARANOVA, O.; HAYASHI, S.; MCMAHON, A.; RAO, M.; PEVNY, L. SOX2, a persistent marker for multipotential neural stem cells derived from embryonic stem cells, the embryo or the adult. Dev. Neurosci., v.26, n, 2-4, p. 148-65, 2004.

ERIKSSO N, P. S.; PERFILIEVA, E.; BJORKERIKSSON, T.; ALBORN, A. M.; NORDBORG, C.; PETERSON, D. A.; GAGE, F. H. Neurogenesis in the adult human hippocampus. Nat. Med., v. 4, p.1313-1317, 1998.

EVERS, B. M.;WEISSMAN, I. L.; FLAKE, A. W.; TABAR, V.; WEISEL, R. D. Stem cells in clinical practice. J. Am. Coll. Surg., v. 197, p. 458-478, 2003. 
FRANKEL, M. S. In search of stem cell policy. Science, v. 298, p. 1397, 2000.

FURUSAWA, C.; KANEKO, K. Theory of robustness of irreversible differentiation in a stem cell system: chaos hypothesis. J. Theor. Biol., v. 209, n.4, p. 395-416, 2001.

GALLAG HER, M.; PELLEYMOUNTER, M. A. Spatial learning deficits in old rats: a model for memory decline in the aged. Neurobiol. Aging, v.9, p. 549-556, 1998.

GOLDMAN, S. A.; NOTTEBOHM, F. Neuronal production, migration, and differentiation in a vocal control nucleus of the adult female canary brain. Proc. Natl. Acad. Sci. USA, v. 80, p. 23902394, 1983.

GOULD, E.; CAMERON, H. A.; DANIELS, D. C.; WO OLLEY, C. S.; MCEWEN, B. S. Adrenal hormones suppress cell division in the adult rat dentate gyrus. J. Neurosci., v.12, p. 3642-3650, 1992.

GOULD, E.; MCEWEN, B. S.; TANAPAT, P.; GALEA, L. A.; FUCHS, E. Neurogenesis in the dentate gyrus of the adult tree shrew is regulated by psychosocial stress and NMDA receptor activation. J. Neurosci., v. 17, p. 2492-2498, 1997.

GOULD, E.; TANAPAT, P.; MCEWEN, B. S.; FLUGGE, G.; FUCHS, E. Proliferation of granule cell precursors in the dentate gyrus of adult monkeys is diminished by stress. Proc. Natl.. Acad. Sci. USA, v.95, p. 3168-3171, 1998.

GOULD, E.; REEVES, A. J.; FALAH, M.; TANAPAT, P.; GROSS, C. G.; FUCHS, E. Hippocampal neurogenesis in adult Old World primates. Proc. Natl. Acad. Sci. USA, v. 96, p. 5263-5267, 1999a

GOULD, E.; REEVES, A. J.; GRAZIANO, M. S.; GROSS, C. G. Neurogenesis in the neocortex of adult primates. Science, v. 286, p.548-552, 1999b.

GOULD, E.; TANAPAT, P.; HASTINGS, N. B.; SHORS, T. J. Neurogenesis in adulthood: a possible role in learning. Trends Cogn. Sci., v. 3, n. 5, p. 186-191, 1999c.

GOULD, E.; BEYLIN, A.; TANAPAT, P.; REEVES, A.; SHORS, T. J. Learning enhances adult neurogenesis in the adult hippocampal formation. Nat. Neurosci., v.2, p.260-265, 1999d.
GUENEAU, G.; PRIVAT, A.; DROUET, J.; COURT, L. Subgranular zone of the dentate gyrus of young rabbits as a secondary matrix. A high-resolution autoradiographic study. Dev. Neurosci., v. 5, p. 345-358, 1982.

KAPLAN, M. S.; HINDS, J. W. Neurogenesis in the adult rat: electron microscopic analysis of light radioautographs. Science, v.197, p. 10921094, 1977.

KEMPERMANN, G.; KUHN, H. G.; GAGE, F. H. Genetic influence on neurogenesis in the dentate gyrus of adult mice. Proc. Natl. Acad. Sci. USA, v. 94, p. 10409-10414, 1997.

KIM, H. T.; KIM, I. S.; LIM, S. E.; LEE, I. S.; PARK, K. I. Gene and cell replacement via neural stem cells. Yonsei Med. J., v.45, Suppl, p. 32-40, 2004.

KORNACK, D. R.; RAKIC, P. Continuation of neurogenesis in the hippocampus of the adult macaque monkey. Proc. Natl. Acad. Sci. USA, v. 96, p. 5768-5773, 1999.

KUHN, H. G.; DICKINSON-ANSON, H.; GAGE, F. H. Neurogenesis in the dentate gyrus of the adult rat: age-related decrease of neuronal progenitor proliferation. J. Neurosci., v.16, p. 20272033, 1996.

KRUGERS, H. J.; DOUMA, B. R.; ANDRINGA, G.; BOHUS, B.; KORF, J.; LUITEN, P. G. Exposure to chronic psychosocial stress and corticosterone in the rat: effects on spatial discrimination learning and hippocampal protein kinase $\mathrm{Cg}$ immunoreactivity. Hippocampus, v. 7, p. 427436, 1997.

LENNINGTON, J. B.; YANG, Z.; CONOVER, J. C. Neural stem cells and the regulation of adult neurogenesis. Reprod. Biol. Endocrinol., v. 1, n. 1, p. 99, 2003.

LOIS, C.; ALVAREZ-BUYLLA, A. Long-distance neuronal migration in the adult mammalian brain. Science, v.264, p. 1145-1148, 1994.

LUSKIN, M. B. Restricted proliferation and migration of postnatally generated neurons derived from the forebrain subventricular zone. Neuron, v.11, p. 173-189, 1993.

MARKAKIS, E. A.; PALMER, T. D.; RANDOLPHMOORE, L.; RAKIC, P.; GAGE, F. H. Novel 
neuronal phenotypes from neural progenitor cells. J. Neurosci., v.24, n. 12, p. 2886-97, 2004.

MILNER, B. Disorders of learning and memory after temporal lobe lesions in man. Clin. Neurosurg., v. 19, p. 421-446, 1972.

MINGER, S. L.; DAVIES, P. Persistent innervation of the rat neocortex by basal forebrain cholinergic neurons despite the massive reduction of cortical target neurons. Morphometric analysis. Exp. Neurol., v. 117, p. 124-138, 1999.

MOGHADDAM, B.; BOLINAO, M. L.; STEINBEHRENS, B.; SAPOLSKY, R. Glucocorticoids mediate the stress-induced extracellular accumulation of glutamate. Brain Res., v. 655, p. 251-254, 1994.

NUNES, M. C.; ROY, N. S.; KEYOUNG, H. M.; GOODMAN, R. R.; MCKHANN, G. 2ND.; JIANG, L.; KANG, J.; NEDERGAARD, M.; GOLDMAN, S. A. Identification and isolation of multipotential neural progenitor cells from the subcortical white matter of the adult human brain. Nat. Med., v. 9, n. 4, p. 439-47, 2003.

NOTTEBOHM, F. A brain for all seasons: cyclical anatomical changes in song control nuclei of the canary brain. Science, v. 214, p. 1368-1370, 1981.

NOTTEBOHM, F. From bird song to neurogenesis. Sci. Am., v. 260, p. 74-79, 1989.

OKANO, H. J.; PFAFF, D. W.; GIBBS, R. B. RB and CDC2 expression in brain: correlations with $3 \mathrm{H}$-thymidine incorporation and neurogenesis. J. Neurosci., v. 13, p. 2930-2938, 1993.

O STENFELD, T.; SVEND SEN, C. N. Recent advances in stem cell neurobiology. Adv. Tech. Stand. Neurosurg., v. 28, p. 83-89, 2003.

PETERSO N, D. A. Stem cell therapy for neurological disease and injury. Panminerva Med., v. 46, n. 1, p. 75-80, 2004.

POTTEN, C. Stem cells in gastrointestinal epithelium: numbers, characteristics and death. Phil. Trans. R. Soc. Lond., v. 353, p. 821-830, 1998.

RAFF, M. Adult stem cell plasticity: fact or artifact? Annu. Rev. Cell Dev. Biol., v. 19, p. 1-22, 2003. REYMONDS, B. A. e WEISS, S. Generation of neurons and astrocytes from isolated cells of the adult mammalian central nervous system. Science, v. 255, p. 5052, p. 1707-10, 1992.

SERI, B.; GARCIA-VERDUGO, J. M.; MCEWEN, B. S.; ALVAREZ-BUYLLA, A. Astrocytes give rise to new neurons in the adult mammalian hippocampus. J. Neurosci., v. 21, p. 7153-7160, 2001.

SHI, Y.; CHICHUNG LIE, D.; TAUPIN, P.; NAKASHIMA, K.; RAY, J.; YU, R. T.; GAGE, F. H.; EVANS, R. M. Expression and function of orphan nuclear receptor TLX in adult neural stem cells. Nature, v. 427, n. 6969, p. 78-83, 2004.

SLACK, J. M. Stem cells in epithelial tissues. Science, v. 287, p. 1431-1433, 2002

SQUIRE, L. R. The neuropsychology of human memory Annu. Rev. Neurosci., v. 5, p. 241273, 1982.

SVEND SEN, C. N.; CALDWELL, M. A.; OSTENFELD, T. Human neural stem cells: isolation, expansion and transplantation. Brain Pathol., v. 9, n. 3, p. 499-513, 1999.

TAUPIN, P.; GAGE, F. H. Adult neurogenesis and neural stem cells of the central nervous system in mammals. J. Neurosci. Res., v. 69, n. 6, p. 745-749, 2002.

TEMPLE, S. The development of neural stem cells. Nature, v. 414, n. 6859, p. 112-7, 2001.

THOMSO N, J. A.; ITSKOVITZ-ELDOR, J.; SHAPIRO, S. S.; WAKNITZ, M. A.; SWIERGIEL, J. J.; MARSHALL, V. S.; JONES, J. M. Embryonic stem cell lines derived from human blastocysts. Science, v. 282, p. 1145-1147, 1998.

TOHILL, M.; TERENGHI, G. Stem-cell plasticity and therapy for injuries of the peripheral nervous system. Biotechnol. Appl. Biochem., v. 40, p. 17-24, 2004.

WAGERS, A. J.; WEISSMAN, I. L. Plasticity of adult stem cells. Cell, v. 116, p. 639-648, 2004.

WATT, F. Epidermal stem cells: markers patterning and the control of stem cell fate. Phil. Trans. R. Soc. Lond., v. 353, p. 831, 1997.

WEBBER, D. J. e MINGER, S. L. Therapeutic potential of stem cells in central nervous system 
regeneration. Curr. Opin. Investig. Drugs, v. 5, n. 7, p. 714-9, 2004.

WEISSMAN, I. L. Translating stem and progenitor cell biology to the clinic: barriers and opportunities. Science, v. 287, p. 1442-1446, 2000.

WESTPHAL, M. e BLACK, P. M. Perspectives of cellular and molecular neurosurgery. J. Neurooncol., v. 70, n. 2, p. 255-69, 2004.
WYSS, J. M e SRIPANIDKULCHAI, B. The development of Ammon's horn and the fascia dentata in the cat a [3H]thymidine analysis. Brain Res., v. 350, p. 185-198, 1985.

ZATZ, M. 0 que é célula tronco? Entrevista à Associação Nacional de Biossegurança (ANBio), Maio 2004. Disponível em <http:// www.anbio.org.br/noticias/celulastronco.htm > Acesso em: 26 maio.2004.

Recebido em/Received in: 06/JUL/2004 Aprovado em/Approved in: 02/SET/2004 